\title{
Varying Constants in Brane World Scenarios
}

\author{
Ph. Brax \\ Service de Physique Théorique \\ CEA-Saclay \\ F-91191, Gif/Yvette cedex, France \\ C. van de Bruck, A. C. Davis, C. S. Rhodes \\ Department of Applied Mathematics and Theoretical Physics \\ Centre for Mathematical Sciences \\ Wilberforce Road, University of Cambridge \\ CB3 0WA, United Kingdom
}

October 29, 2013

\begin{abstract}
Higher-dimensional theories imply that some constants, such as the gravitational constant and the strength of the gauge-couplings, are not fundamental constants. Instead they are related to the sizes of the extra-dimensional space, which are moduli fields in the fourdimensional effective theory. We study the cosmological evolution of the moduli fields appearing in brane world scenarios and discuss the implications for varying constants.
\end{abstract}

\section{Introduction}

Theories based on the idea of extra dimensions predict the existence of scalar fields (also called moduli fields), which are related to the size of the higher-dimensional space. Classically these fields do not have a potential, but they might acquire one due to supersymmetry breaking or quantum mechanical effects. However, a potential does not necessarily guarantee stabilization of the fields.

From the phenomenological point of view there are motivations to study the cosmological evolution of moduli fields. First, it is well known that in scalar-tensor theories of gravity there exists an attractor mechanism which drives the theory towards general relativity [1]. Thus, it might well be that such attractor solutions exist for moduli fields in higher dimensional theories as well, which would drive the theory towards those points in moduli space which are consistent with observations. Another motivation is the claim of a varying fine-structure constant made in [2]. If this observation is confirmed, this would point towards a theory in which some of the moduli fields are not stabilised but are dynamical at least during some part of the cosmological history.

In this paper we consider a class of brane world models and the cosmological evolution of moduli fields. In particular we discuss which constants necessarily vary in the brane world context. We begin with a description of the effective theory in four dimensions.

\section{The Effective Theory at Low Energies}

We consider a five-dimensional theory with two branes and a bulk scalar field. The bulk scalar field induces tension on each branes with the same amplitude but with different signs $\left(U_{1}=-U_{2}\right)$ (see Figure 1). 


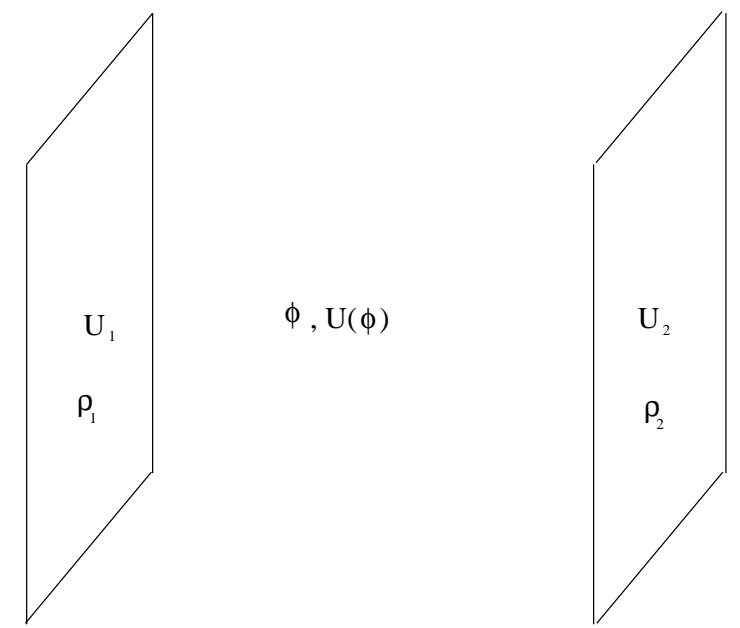

Figure 1: The five-dimensional setup consists of two boundary branes and a bulk scalar field with bulk potential $U$. Furthermore, the scalar field induce a brane tension on each branes with opposite signs.

At low energies, the action can be obtained using the moduli space approximation and we found [3] that the theory can be described by the following action (written here in the Einstein frame):

$$
\begin{aligned}
S_{\mathrm{EF}} & =\frac{1}{16 \pi G} \int d^{4} x \sqrt{-g}\left[\mathcal{R}-\frac{12 \alpha^{2}}{1+2 \alpha^{2}} \frac{(\partial Q)^{2}}{Q^{2}}-\frac{6}{2 \alpha^{2}+1}(\partial R)^{2}\right] \\
& -\int d^{4} x \sqrt{-g}\left(V_{\mathrm{eff}}(Q, R)+W_{\mathrm{eff}}(Q, R)\right) \\
& -S_{m}^{(1)}\left(\Psi_{1}, A^{2}(Q, R) g_{\mu \nu}\right)-S_{m}^{(2)}\left(\Psi_{2}, B^{2}(Q, R) g_{\mu \nu}\right) .
\end{aligned}
$$

In this expression, $Q$ and $R$ are two scalar degrees of freedom with potential energies $V_{\text {eff }}$ and $W_{\text {eff. }} S_{m}^{(i)}$ denote the matter action for each matter types.

The cosmological evolution equations can found to be:

$$
H^{2}=\frac{8 \pi G}{3}\left(\rho_{1}+\rho_{2}+V_{\text {eff }}+W_{\text {eff }}\right)+\frac{2 \alpha^{2}}{1+2 \alpha^{2}} \dot{\phi}^{2}+\frac{1}{1+2 \alpha^{2}} \dot{R}^{2} .
$$

where we have defined $Q=\exp \phi$. The field equations for $R$ and $\phi$ read

$$
\begin{aligned}
\frac{6}{1+2 \alpha^{2}}[\ddot{R}+3 H \dot{R}] & =-8 \pi G\left[\frac{\partial V_{\text {eff }}}{\partial R}+\frac{\partial W_{\text {eff }}}{\partial R}\right. \\
+ & \left.2 \alpha_{R}^{(1)}\left(\rho_{1}-3 p_{1}\right)+2 \alpha_{R}^{(2)}\left(\rho_{2}-3 p_{2}\right)\right] \\
\ddot{\phi}+3 H \dot{\phi}= & -8 \pi G \frac{1+2 \alpha^{2}}{12 \alpha^{2}}\left[\frac{\partial V_{\text {eff }}}{\partial \phi}\right. \\
+ & \left.\frac{\partial W_{\text {eff }}}{\partial \phi}+2 \alpha_{\phi}^{(1)}\left(\rho_{1}-3 p_{1}\right)+2 \alpha_{\phi}^{(2)}\left(\rho_{2}-3 p_{2}\right)\right] .
\end{aligned}
$$

Finally, the coupling parameters are given by

$$
\begin{aligned}
& \alpha_{\phi}^{(1)}=-\frac{2 \alpha^{2}}{1+2 \alpha^{2}}, \quad \alpha_{\phi}^{(2)}=-\frac{2 \alpha^{2}}{1+2 \alpha^{2}}, \\
& \alpha_{R}^{(1)}=\frac{\tanh R}{1+2 \alpha^{2}}, \quad \alpha_{R}^{(2)}=\frac{(\tanh R)^{-1}}{1+2 \alpha^{2}} .
\end{aligned}
$$




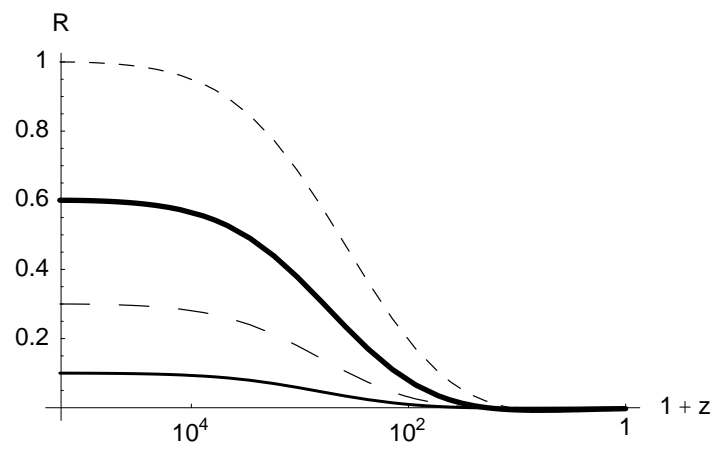

Figure 2: Evolution of the field $R$ during radiation and matter dominated epochs for the case of no matter on the negative tension brane.

There is an obervational constraint for models described by an action like the one above [4], namely the parameter $\theta$, defined by

$$
\theta=\frac{4}{3} \frac{\alpha^{2}}{1+2 \alpha^{2}}+\frac{\tanh ^{2} R}{6\left(1+2 \alpha^{2}\right)}
$$

has to be less than $10^{-3}$. This implies, that

$$
\alpha<10^{-2}, R<0.2 .
$$

The question is, therefore, whether $R$ is driven towards small values during the cosmological evolution. Furthermore, even if such an attractor exists, it is not clear if the attractor is efficient enough. To find an answer to these questions, we have numerically solved the equations of motions for different cases, which we will describe in the next section.

\section{Cosmological Evolution}

There are different cases to study. For example, there could be no matter on the negative tension brane or no potentials for the fields. The equations of motion of $R$ and $\phi$ show that their evolution depends on the matter content on the branes. We will assume both radiation and matter on the positive tension brane and will follow the evolution of the fields during the radiation and matter dominated epoch for the cases without and with matter on the negative tension brane.

\subsection{No matter on the negative tension brane}

We assume no potential energy for the fields $R$ and $\phi$. In this case, the evolution of $R$ and $\phi$ are shown in fig. 2 and fig. 3.

During the radiation dominated epoch, the fields $\phi$ and $R$ do not evolve strongly. This is because any time-evolution is sourced by the trace of the energy-momentum tensor, which vanishes if radiation dominates the expansion. During matter domination, one can clearly see that $R$ is driven towards small values for a wide range of initial conditions. Also, the evolution of $\phi$ does not strongly depend on the initial conditions. Thus, we conclude that there is an attractor for the field $R$, when there is no matter on the second brane.

\subsection{Including matter on the negative tension brane}

We still assume no potential energy for the fields $R$ and $\phi$. The evolution of $R$ and $\phi$ are shown in figs. 4 and 5. 


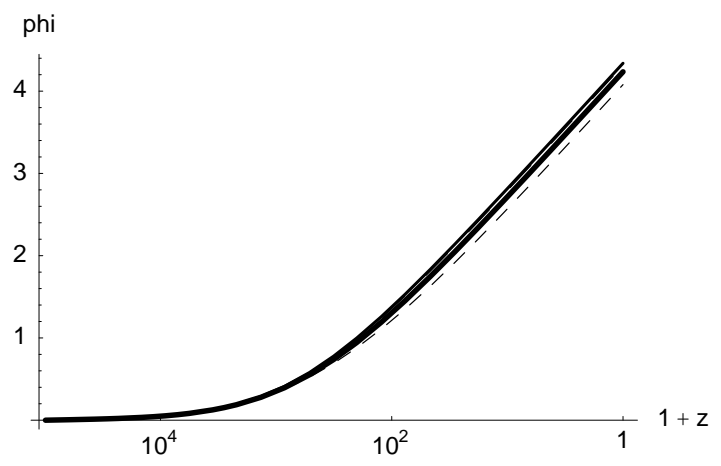

Figure 3: Evolution of the field $\phi$ during radiation and matter dominated epochs for the case of no matter on the negative tension brane.

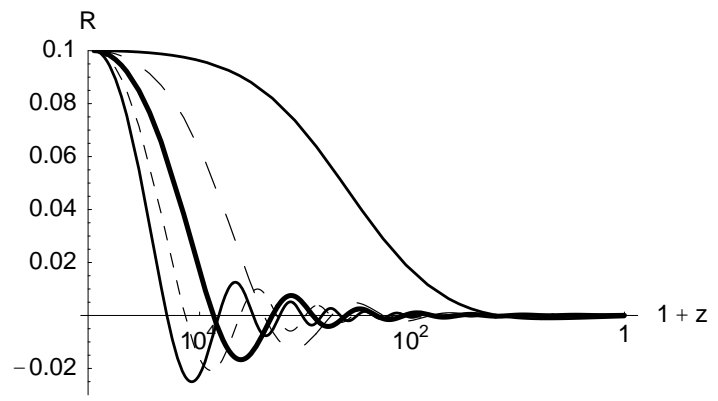

Figure 4: Evolution of the field $R$ during radiation and matter dominated epochs for the case with matter on the negative tension brane.

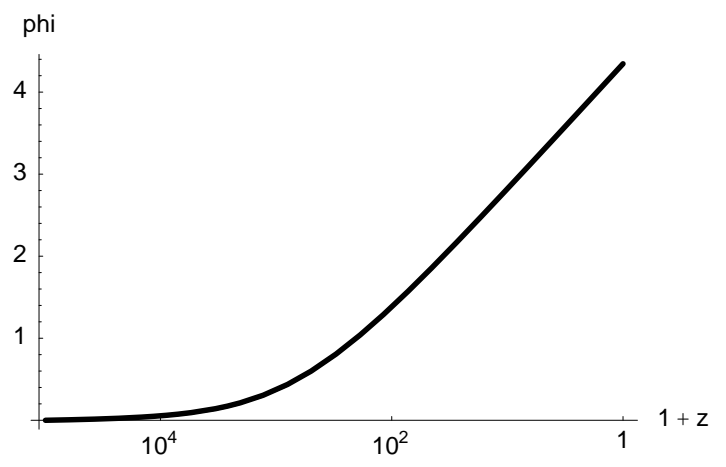

Figure 5: Evolution of the field $\phi$ during radiation and matter dominated epochs for the case with matter on the negative tension brane. 
We see, that in this case, the attractor is more effective. Thus, in both cases, the field $R$ is driven towards small values. In [3] we have also included potentials for the fields, which might be necessary for the theory to be consistent. We refer to [3] for more details.

\section{Implications for varying constants}

In the Einstein frame, masses of particles vary, because the two scalar fields $\phi$ and $R$ couple to the matter on both branes. Alternatively, it is possible to consider the frame in which the masses of particles living on the positive tension brane are constant; in that case, the gravitational constant is time-varying as well as the masses of particles confined on the negative tension brane. These conclusions are generic for the kind of theories we consider. The constraint (8) ensures that the time-variation is consistent with current observations. (There are constraints on the amplitude of $R$ and $\phi$ in the early universe from nucleosynthesis. We do not discuss this issue here.)

Is there any time variation for the electromagnetic fine structure constant? As explained in [3], this is not the case, as long as vector bosons are not coupled to the bulk scalar field (and therefore to $\phi$ and $R$ ). Thus, what is needed in order to explain the variation of the fine-structure constant is a coupling of the form

$$
S_{m}=-\frac{1}{4 g^{2}} \int \sqrt{-g_{B}} f(R, \phi) g_{B}^{\mu \rho} g_{B}^{\nu \eta} F_{\mu \rho} F_{\nu \eta}
$$

Such a coupling appears naturally in heterotic M-theory, for example [5]. In this theory the bulk scalar field has an geometrical interpretation: it is a measure of the deformation of a Calabi-Yau threefold. Generally, one would expect that the function $f$ is a function of the parameter $\alpha$ and that for $\alpha \rightarrow 0$ one has $f \rightarrow 1$, because for $\alpha=0$ the bulk scalar field decouples from the dynamics [3]. The attractor behaviour found in the last section implies, that any time-variation of the gauge-couplings at redshift between 0 and 5 is mainly due to the evolution of $\phi$. The amount of variation depends on the function $f$ above.

To conclude, the attractor behaviour we have identified might be related to the stabilization of the moduli fields in brane world scenarios. When the moduli fields are not stabilised, an evolution of masses or the gravitational constant (depending on the frame) is generally predicted, whereas an evolution of the fine-structure constant is not generically predicted. Only if vector bosons are directly coupled to the bulk scalar field one would expect coupling constants to vary. This is the

case in models based on string theory and therefore one would necessary predict a time-variation of coupling constants as long as the moduli fields are not stabilized at a minimum of some potential.

Acknowledgements: C.v.d.B. is grateful to Malcolm Fairbairn for useful discussions on varying constants in M-theory. This work was supported by PPARC (C.v.d.B., A.-C.D. and C.S.R.), a CNRS-Royal Society exchange grant for collaborative research and the European network (RTN), HPRN-CT-200-00148 and PRN-CT-2000-00148.

\section{References}

[1] T. Damour and K. Nordtvedt, Phys.Rev.Lett. 70, 2217 (1993)

[2] J.K. Webb et al, Phys.Rev.Lett. 87, 091301 (2001)

[3] Ph. Brax, C. van de Bruck, A.-C. Davis and C.S. Rhodes, Cosmological Evolution of Brane World Moduli, hep-th/0209158

[4] T. Damour and G. Esposito-Farese, Class.Quant.Grav. 9, 2093 (1992)

[5] E. Witten, Nucl.Phys. B471, 135 (1996); A. Lukas, B.A. Ovrut and D. Waldram, Nucl.Phys.B 532, $43(1998)$ 